\title{
Direct mediation of metastable supersymmetry breaking
}

\author{
Ryuichiro Kitano, ${ }^{1,2}$ Hirosi Ooguri, ${ }^{3}$ and Yutaka Ookouchi ${ }^{3}$ \\ ${ }^{1}$ Stanford Linear Accelerator Center, Stanford University, Stanford, California 94309, USA \\ ${ }^{2}$ Physics Department, Stanford University, Stanford, California 94305, USA \\ ${ }^{3}$ California Institute of Technology, Pasadena, California 91125, USA
}

(Received 20 December 2006; published 28 February 2007)

\begin{abstract}
The supersymmetric $\mathrm{SU}\left(N_{C}\right)$ Yang-Mills theory coupled to $N_{F}$ matter fields in the fundamental representation has metastable vacua with broken supersymmetry when $N_{C}<N_{F}<\frac{3}{2} N_{C}$. By gauging the flavor symmetry, this model can be coupled directly to the standard model. We show that it is possible to make a slight deformation to the model so that gaugino masses are generated and the Landau pole problem can be avoided. The deformed model has simple realizations on intersecting branes in string theory, where various features of the metastable vacua are encoded geometrically as brane configurations.
\end{abstract}

DOI: $10.1103 /$ PhysRevD.75.045022

PACS numbers: 11.30.Pb, 11.30.Qc, 12.60.Jv

\section{INTRODUCTION}

Although there is no clear evidence yet, it is plausible that softly broken $\mathcal{N}=1$ supersymmetry is realized in nature. Not only because it is a symmetry possessed by string theory; there are many phenomenologically attractive features in supersymmetric models, such as cancellation of quadratic divergences and unification of the gauge coupling constants [1-3].

It is then a question of how supersymmetry is broken and how we feel it. There have been many studies on this subject, but, as is often the case, one of the earliest proposals $[4,5]$ among them seems to be the most elegant and simple idea. The idea is that there is a QCD-like strong interaction which breaks supersymmetry dynamically, and the standard model gauge group is identified with a subgroup of flavor symmetry in this sector. The standard model gauge sector can, therefore, feel the supersymmetry breaking directly via one-loop diagrams.

This idea has been discarded for a long time because of its difficulty in realistic model building. First, Witten has shown that there is a supersymmetric vacuum in supersymmetric QCD by using an index argument [6]. Therefore, we are forced to think of the possibility of chiral gauge theories for supersymmetry breaking, which is already a bit complicated. (See [7-9] for dynamical supersymmetry breaking in chiral gauge theories, and $[10,11]$ for models of direct gauge mediation in that context.) There is also a problem of Landau poles of the standard model gauge interactions. Once we embed the gauge group of the standard model into a flavor group of the dynamical sector (this itself is not a trivial task), many particles appear which transform under the standard model gauge group. These fields contribute to beta functions of the gauge coupling constants and drive them to a Landau pole below the unification scale. Finally, even though the gauge sector of the standard model directly couples to the supersymmetry breaking dynamics, it is nontrivial whether or not we can obtain the gaugino masses. It is often the case that the leading contribution to gaugino masses cancels out.
Very recently, there was a breakthrough on this subject. Intriligator, Seiberg, and Shih (ISS) have shown that there is a metastable supersymmetry breaking vacuum in some of the supersymmetric QCD theories [12]. The model is simply $\mathrm{SU}\left(N_{C}\right)$ gauge theory with massive (but light) $N_{F}$ quarks. Within a range $N_{C}<N_{F}<\frac{3}{2} N_{C}$, supersymmetry is broken in the metastable vacuum. The possibility of direct gauge mediation in this model is also discussed in Ref. [12]. ${ }^{1}$ Because of the simplicity of the model, it is straightforward to embed the standard model gauge group into the $\mathrm{SU}\left(N_{F}\right)$ flavor symmetry. However, it is concluded that there are still problems regarding the Landau pole and the gaugino masses. In the ISS model, there is an unbroken approximate $\mathrm{U}(1)_{R}$ symmetry which prevents us from obtaining the gaugino masses.

The $\mathrm{U}(1)_{R}$ problem is a common feature in models of gauge mediation. As is discussed recently in Ref. [14], if the low energy effective theory of the dynamical supersymmetry breaking model is of the O'Raifeartaigh type, there is an unbroken $R$-symmetry at the minimum of the potential (the origin of the field space). It has been proposed that the inverted hierarchy mechanism [15] can shift the minimum away from the origin by the effect of gauge interactions [14,16-19]. An alternative possibility that the shift is induced by an $R$-symmetry breaking term in supergravity Lagrangian (the constant term in the superpotential) is recently discussed in Ref. [20]. It is, however, still nontrivial whether we obtain the gaugino masses even with the $R$-symmetry breaking vacuum expectation values in direct gauge mediation models. For example, a model in Ref. [21] generates gaugino masses only at the $F^{3}$ order even though the $R$-symmetry is broken by assuming the presence of the local minimum away from the origin. Since the scalar masses squared are obtained at the $F^{2}$ order as usual, gaugino masses are much smaller than the scalar masses unless the messenger scale is $O(10 \mathrm{TeV})$, that is difficult in models of direct gauge mediation because of the

\footnotetext{
${ }^{1}$ See also [13] for a related work.
} 
Landau pole problem. In fact, as we will see later, the structure of the messenger particles in the ISS model is the same as that in this model. (The same structure can be found in many models, for example, in Ref. [22] and also in very early proposals of gauge mediation models in Refs. [23-25].) Therefore, it is not sufficient to destabilize the origin of the field space for generating both gaugino and scalar masses.

In this paper we propose a slight deformation to the ISS model with which we can obtain gaugino masses by identifying a flavor subgroup with the standard model gauge group. We add a superpotential term which breaks $R$-symmetry explicitly so that nonvanishing gaugino masses are induced. The vacuum structure becomes richer by the presence of the new term. In addition to the vacuum that is obtained by a slight perturbation to the ISS metastable vacuum, which we will call the ISS vacuum, there appear new (but phenomenologically unacceptable) metastable vacua. We find that decays of the ISS vacuum into the other vacua are sufficiently slow so that it is phenomenologically viable.

We also show that the Landau pole problem can be avoided by keeping the dynamical scale of the ISS sector sufficiently high in a way that is compatible with phenomenological requirements. In addition, if metastable vacua exist in a model with the same number of colors and flavors, as suggested by ISS, we can also consider the case where the ISS sector is in the conformal window, $\frac{3}{2} N_{C} \leq N_{F}<3 N_{C}$. In this case, we can take the scales of the ISS sector as low as $O(100-1000 \mathrm{TeV})$.

The deformed ISS model can be realized on intersecting branes in string theory, where a rich vacuum structure and the metastability of vacua can be understood geometrically.

\section{THE ISS MODEL}

We first review the ISS model. The model is simply a supersymmetric QCD with light flavors. Perturbative corrections to a scalar potential are calculable in the magnetic dual picture, and they have been found to stabilize a supersymmetry breaking vacuum. The model has an unbroken $R$-symmetry, which prevents it from generating gaugino masses. An explicit one-loop computation of the masses suggests a natural solution to this problem, which we will discuss in the next section.

\section{A. Supersymmetry breaking}

The model is an $\mathrm{SU}\left(N_{C}\right)$ gauge theory with $N_{F}$ flavors. The quarks have mass terms:

$$
W=m_{i} Q_{i} \bar{Q}_{i} .
$$

The index $i$ runs for $i=1, \ldots, N_{F}$. The masses $m_{i}$ are assumed to be much smaller than the dynamical scale $\Lambda$. There is a metastable supersymmetry breaking vacuum when $N_{C}<N_{F}<\frac{3}{2} N_{C}$, where there is a weakly coupled description of the theory below the dynamical scale $\Lambda$. The gauge group of the theory is $\mathrm{SU}\left(N_{F}-N_{C}\right)$ and degrees of freedom at low energy are meson fields $M_{i j} \sim Q_{i} Q_{j}$ and dual quarks $q_{i}$ and $\bar{q}_{i}$. There are superpotential terms:

$$
W=m_{i} M_{i i}-\frac{1}{\hat{\Lambda}} q_{i} M_{i j} \bar{q}_{j}
$$

A dimensionful parameter $\hat{\Lambda}$ is introduced so that the dimensionality of the superpotential is correct. A natural scale of $\hat{\Lambda}$ is $O(\Lambda)$.

With this superpotential, the $F_{M}=0$ condition for all components of $M_{i j}$ cannot be satisfied. The rank of the matrix $q_{i} \bar{q}_{j}$ is at most $N_{F}-N_{C}$ whereas the mass matrix $m_{i}$ has the maximum rank, $N_{F}$. The lowest energy vacuum is at

$$
M_{i j}=0, \quad q_{i}=\bar{q}_{i}=\left(\begin{array}{c}
\sqrt{m_{I} \hat{\Lambda}} \delta_{I J} \\
0
\end{array}\right),
$$

where $I$ and $J$ runs from 1 to $N_{F}-N_{C}$, and $m_{i}$ is sorted in descending order. The $F$-components of $M_{i i}$ with $i=$ $N_{F}-N_{C}+1, \ldots, N_{F}$ have nonvanishing value $m_{i}$. At this vacuum, the gauge symmetry $\mathrm{SU}\left(N_{F}-N_{C}\right)$ is completely broken.

We parametrize fluctuations around this vacuum to be:

$$
\frac{\delta M_{i j}}{\hat{\Lambda}}=h\left(\begin{array}{cc}
Y_{I J} & Z_{I a} \\
\tilde{Z}_{a I} & \hat{\Phi}_{a b}
\end{array}\right), \quad \delta q_{i}=\left(\begin{array}{c}
\chi_{I J} \\
\rho_{I a}
\end{array}\right), \quad \delta \bar{q}_{i}=\left(\begin{array}{c}
\tilde{\chi}_{I J} \\
\tilde{\rho}_{I a}
\end{array}\right) .
$$

We put dimensionless parameter $h$ of $O(1)$ so that components have a canonically normalized kinetic term. Again, $I, J=1, \ldots, N_{F}-N_{C}$ and $a, b=1, \cdots, N_{C}$. Among these fields $\hat{\Phi}_{a b}$ and the trace part of $\chi-\tilde{\chi}, \operatorname{Tr}[\chi-\tilde{\chi}] \equiv$ $\operatorname{Tr} \delta \hat{\chi}$, remains massless at tree level. The other fields obtain masses of $O(\sqrt{m \Lambda})$. A one-loop correction to a potential for the pseudomoduli $\hat{\Phi}$ and $\operatorname{Re}[\operatorname{Tr} \delta \hat{\chi}]$ is shown to give positive masses squared, which ensures the stability of the vacuum. ${ }^{2}$ Once we take into account the nonperturbative effect, the true supersymmetric vacuum appears far away from the origin of the meson field $M$. The lifetime of the false vacuum can be arbitrarily long if $m_{i} \ll \Lambda$. Also, interestingly, the supersymmetry breaking vacuum is preferred in the thermal history of the Universe [26-28].

\section{B. Gaugino masses}

It is possible to embed the standard model gauge group into a flavor symmetry group of this model. When we take $m_{1}=\ldots=m_{N_{F}-N_{C}}=m$ and $m_{N_{F}-N_{C}+1}=\ldots=m_{N_{F}}=$ $\mu$, there is a global symmetry; $\mathrm{SU}\left(N_{F}-N_{C}\right)_{F} \times$ $\mathrm{SU}\left(N_{C}\right)_{F} \times \mathrm{U}(1)_{B}$. With $N_{F}-N_{C} \geq 5$ or $N_{C} \geq 5$, we

\footnotetext{
${ }^{2}$ The imaginary part of $\operatorname{Tr} \delta \hat{\chi}$ is a Goldstone boson associated with a broken $\mathrm{U}(1)_{B}$ symmetry.
} 
can embed $\mathrm{SU}(3) \times \mathrm{SU}(2) \times \mathrm{U}(1)$ into $\mathrm{SU}\left(N_{F}-N_{C}\right)_{F}$ or $\mathrm{SU}\left(N_{C}\right)_{F}$, respectively. In the case where we embed $\mathrm{SU}(3) \times \mathrm{SU}(2) \times \mathrm{U}(1)$ into the $\mathrm{SU}\left(N_{F}-N_{C}\right)_{F}$ flavor symmetry, the standard model gauge group at low energy is a diagonal subgroup of $\mathrm{SU}(3) \times \mathrm{SU}(2) \times \mathrm{U}(1)$ in $\mathrm{SU}\left(N_{F}-N_{C}\right)$ dual gauge interaction (under which $q$ and $\bar{q}$ transform and $M$ is neutral) and that in the $\mathrm{SU}\left(N_{F}-\right.$ $\left.N_{C}\right)_{F}$ flavor group.

As discussed in Ref. [12], there is an unbroken $R$-symmetry under which $M$ carries charge two and $q$ and $\bar{q}$ are neutral. Since the $R$-symmetry forbids the gaugino masses, there is no contribution to the gaugino masses of the standard model gauge group even though it is directly coupled to a supersymmetry breaking sector. It is instructive to see how the gaugino masses vanish at one loop. The fields $\rho$ and $\tilde{\rho}$ carry quantum numbers of both $\mathrm{SU}\left(N_{F}-N_{C}\right)$ and $\mathrm{SU}\left(N_{C}\right)_{F}$ and couple to $\hat{\Phi}$ which has nonvanishing vacuum expectation value in the $F$-component. Therefore $\rho$ and $\tilde{\rho}$ play a role of messenger fields in gauge mediation. ${ }^{3}$ The relevant superpotential for this discussion is

$$
W=-h \rho \hat{\Phi} \tilde{\rho}-h \bar{m}(\rho \tilde{Z}+\tilde{\rho} Z),
$$

where we suppressed indices and defined $\bar{m} \equiv \sqrt{m \hat{\Lambda}}$. The $\rho$ and $Z$ fields have mixing terms. In a matrix notation,

$$
W=h(\rho, Z) \mathcal{M}\left(\begin{array}{l}
\tilde{\rho} \\
\tilde{Z}
\end{array}\right),
$$

where $\mathcal{M}$ is a mass matrix for the messenger fields

$$
\mathcal{M}=\left(\begin{array}{cc}
\hat{\Phi} & \bar{m} \\
\bar{m} & 0
\end{array}\right) .
$$

The formula for the gaugino masses can be generalized for this multimessenger case as follows:

$$
m_{\lambda}=\frac{g^{2} \bar{N}}{(4 \pi)^{2}} F_{\hat{\Phi}} \frac{\partial}{\partial \hat{\Phi}} \log \operatorname{det} \mathcal{M},
$$

where $\bar{N}$ is $N_{C}$ or $N_{F}-N_{C}$ depending on whether we embed the standard model gauge group into the $\mathrm{SU}\left(N_{F}-\right.$ $\left.N_{C}\right)_{F}$ or the $\mathrm{SU}\left(N_{C}\right)_{F}$ flavor symmetry. This formula is valid when $F_{\hat{\Phi}} \ll \bar{m}^{2}$. Since there is no $\hat{\Phi}$ dependence in $\operatorname{det} \mathcal{M}$, we obtain $m_{\lambda}=0$.

We can now clearly see that the gaugino mass would vanish at the leading order in $F_{\Phi} / \bar{m}^{2}$ even if we could obtain a nonvanishing vacuum expectation value for $\hat{\Phi}$ which breaks the $R$-symmetry [21]. In the following section, we consider a model with explicit $R$-symmetry breaking which generates the gaugino masses at the leading order in $F_{\Phi} / \bar{m}^{2}$.

\footnotetext{
${ }^{3}$ The standard model gauge group at low energy partly comes from $\mathrm{SU}\left(N_{F}-N_{C}\right)$ when we embed the $\mathrm{SU}(3) \times \mathrm{SU}(2) \times \mathrm{U}(1)$ into $\mathrm{SU}\left(N_{F}-N_{C}\right)_{F}$. One-loop diagrams with the $\rho$ and $\tilde{\rho}$ fields, therefore, contribute to the gaugino masses also in this case, although they are not charged under $\mathrm{SU}\left(N_{F}-N_{C}\right)_{F}$.
}

\section{DEFORMED ISS MODEL}

Motivated by discussion in the previous section, we consider a modification of the ISS model which contains a mass term for the meson fields $Z$ and $\tilde{Z}$ so that $\operatorname{det} \mathcal{M}$ has $\hat{\Phi}$ dependence. In the electric description, this corresponds to adding the following superpotential term

$$
W \ni-\frac{1}{m_{X}}\left(Q_{I} \bar{Q}_{a}\right)\left(Q_{a} \bar{Q}_{I}\right),
$$

where the color $\mathrm{SU}\left(N_{C}\right)$ indices are contracted in $(Q \bar{Q})$. Though this is a nonrenormalizable interaction, it can be generated by integrating out extra massive fields coupled to $\left(Q_{a}, Q_{I}\right)$ in a renormalizable theory. In Sec. IV, we will show that such a theory can be realized on intersecting branes in string theory. This interaction preserves the global symmetry $\mathrm{SU}\left(N_{F}-N_{C}\right)_{F} \times \mathrm{SU}\left(N_{C}\right)_{F} \times \mathrm{U}(1)_{B}$. We assume the same structure for mass terms of $Q$ and $\bar{Q}$ as that in the model in the previous section, i.e.,

$$
W_{\text {mass }}=m\left(Q_{I} \bar{Q}_{I}\right)+\mu\left(Q_{a} \bar{Q}_{a}\right),
$$

so that the global symmetry is preserved. In the magnetic description, the mass terms correspond

$$
W_{\text {mass }}^{\text {mag. }}=\bar{m}^{2} \operatorname{Tr} Y+\bar{\mu}^{2} \operatorname{Tr} \hat{\Phi},
$$

where $\bar{m}^{2} \equiv m \hat{\Lambda}$ and $\bar{\mu}^{2} \equiv \mu \hat{\Lambda}$. In terms of component fields, the full superpotential is given by

$$
\begin{aligned}
W= & h \operatorname{Tr}\left[\bar{m}^{2} Y+\bar{\mu}^{2} \hat{\Phi}-\chi Y \tilde{\chi}-\chi Z \tilde{\rho}\right. \\
& \left.-\rho \tilde{Z} \tilde{\chi}-\rho \hat{\Phi} \tilde{\rho}-m_{z} Z \tilde{Z}\right] .
\end{aligned}
$$

We could have added other terms compatible with the global symmetry. Although the theorem of [29] implies that a generic deformation to the superpotential generates a supersymmetry preserving vacuum at tree level, it may not cause a problem with our scenario as far as the new vacuum is far from the one we are interested in and the transition rate between the vacua is small. However, since there are tree-level flat directions in $\hat{\Phi}$, a deformation by $\operatorname{Tr} \hat{\Phi}^{2}$ destabilizes the ISS vacuum. Whether such a deformation is prohibited is a question of ultraviolet completions of the theory, but there is an interesting observation we can make from the point of view of the low energy effective theory. As we will see later, we need a certain level of hierarchy between $m$ and $\mu(\mu \ll m)$ to suppress a tunneling rate into unwanted vacua and also to avoid a Landau pole of the gauge coupling of the standard model gauge interaction. With this hierarchy this model possesses an approximate (anomalous) $R$-symmetry which is softly broken by the small mass term $\mu$. The charge assignment is $R\left(Q_{I}\right)=$ $R\left(\bar{Q}_{I}\right)=1$ and $R\left(Q_{a}\right)=R\left(\bar{Q}_{a}\right)=0$. This symmetry justifies the absence or suppression of other higher dimensional operators such as $\operatorname{Tr} \hat{\Phi}^{2}$ which destabilize the supersymmetry breaking vacua. (The supersymmetry 
breaking vacua remain stable as far as the coefficient for $\operatorname{Tr} \hat{\Phi}^{2}$ is smaller than $\mu$.)

\section{A. Vacuum structure}

The introduction of the mass term for $Z$ and $\tilde{Z}$ makes the vacuum structure of this model quite rich. In addition to the supersymmetric and supersymmetry breaking vacua in the ISS model, there are also several stable supersymmetry breaking vacua. The stability and decay probability between these vacua are controlled by parameters in superpotential.

\section{Metastable supersymmetry breaking vacua}

As long as $m_{z}$ is smaller than $\bar{m}$, we can think of $m_{z}$ as a small perturbation to the ISS model, and thus there exists a similar metastable supersymmetry breaking vacuum. The effect of a finite value of $m_{z}$ is a small shift of $\hat{\Phi}$ of $O\left(m_{z}\right)$. The pseudomoduli $\hat{\Phi}$ and $\operatorname{Re}[\operatorname{Tr} \delta \hat{\chi}]$ obtain masses of $O\left(h^{2} \bar{\mu}^{2} / \bar{m}\right)$ as in the ISS model. We show in Fig. 1 the one-loop effective potential for the pseudomoduli $\hat{\Phi}$. We see a small shift of the minimum. For $m_{z}>\bar{m}$, this vacuum is destabilized. Therefore, we assume in the following that $m_{z}$ is smaller than $\bar{m}$.

The small $m_{z}$, in fact, modifies the vacuum structure drastically far away from the origin of the field space. We can find other supersymmetry breaking vacua with

$$
\begin{gathered}
\rho \tilde{\rho}=\frac{m_{z}^{2}}{m^{2}} Z \tilde{Z}=\operatorname{diag}\left(\bar{\mu}^{2}, \ldots \bar{\mu}^{2}, 0 \ldots 0\right), \\
\chi \tilde{\chi}=\bar{m} \mathbf{1}_{N_{F}-N_{C}}, \\
Y=-\frac{\bar{\mu}^{2}}{m_{z}} \mathbf{1}_{N_{F}-N_{C}}, \quad \hat{\Phi}=-\frac{\bar{m}^{2}}{m_{z}} \operatorname{diag}(1, \ldots 1,0, \ldots 0), \\
V_{\text {lower }}=\left(N_{C}-n\right)\left|h \bar{\mu}^{2}\right|^{2},
\end{gathered}
$$

where the number of $\bar{\mu}^{2}$ in the first equation, denoted $n$,

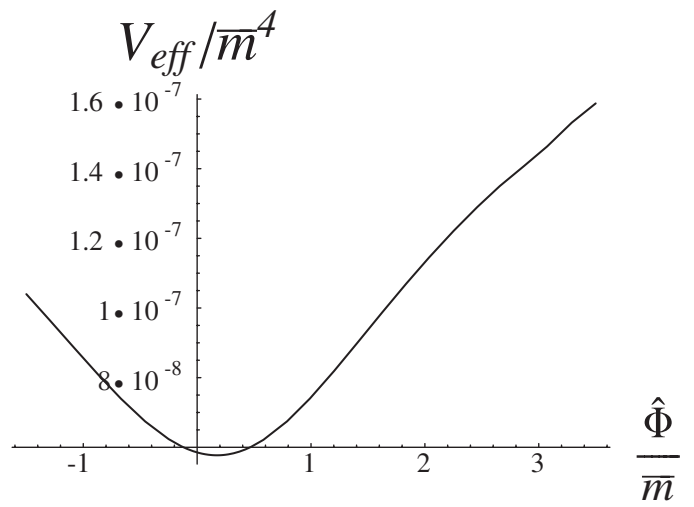

FIG. 1. One-loop effective potential $V_{\text {eff }}(\hat{\Phi} / \bar{m})$ along the real axis of $\hat{\Phi}$ for $\operatorname{Re}[\operatorname{Tr} \delta \hat{\gamma}]=0, m_{z}=\bar{m} / 3$, and $\mu=\bar{m} / 100$. The critical point is at $\hat{\Phi}=0.1747 \bar{m}$. At $\hat{\Phi} \sim 3 \bar{m}$ there is a tachyonic direction toward nonzero $\rho, \tilde{\rho}, Z$, and $\tilde{Z}$ in field configuration space. runs from 1 to $N_{F}-N_{C}$. Since these vacua have energy that are lower than that of the ISS vacua, $V_{\text {ISS }}=N_{C}\left|h \bar{\mu}^{2}\right|^{2}$, it has nonzero transition probability to these vacua. Below, we show that the decay rate can be made parametrically small by a mass hierarchy, $\bar{\mu} \ll \bar{m}$. Although the vacuum with $n=N_{F}-N_{C}$ is the global minimum of the classical potential, they are not phenomenologically viable since gauginos cannot get masses at the leading order in $F / \bar{m}^{2}$ for the same reason as in the original ISS model when we embed the standard model into some of unbroken global symmetry.

As we have seen, our vacuum is not the global minimum of the potential. It can decay into lower energy vacua specified by (13) and (14). We estimate the decay rate by evaluating the Euclidean action from our vacuum to others. The barrier by the one-loop potential is not high, of order $O\left(\bar{\mu}^{4}\right)$. Thus, the most efficient path is to climb up the potential of $\hat{\Phi}$ and then slide down to more stable supersymmetry breaking vacua. The distance between $\left.\langle\hat{\Phi}\rangle\right|_{\text {lower }}$ and $\left.\langle\hat{\Phi}\rangle\right|_{\text {ISS }}$ is of order $O\left(\bar{m}^{2} / m_{z}\right)$ and is wide compared to the height of the potential. Thus, we can estimate bounce action with triangle approximation [30],

$$
S \sim\left(\frac{\bar{m}}{\bar{\mu}}\right)^{4}\left(\frac{\bar{m}}{m_{z}}\right)^{4} .
$$

Even if we choose $m_{z} \sim \bar{m}$, which will be required below, the Euclidean action can be made parametrically large by taking $\bar{\mu} \ll \bar{m}$. Thus, the decay rate is parametrically small.

One might think that we can find a more efficient path through tree-level potential barrier. However at least it has to climb up $V_{\text {peak }} \sim O\left(\bar{\mu}^{2} \bar{m}^{2}\right)$ that is very high, compared to the difference between two supersymmetry breaking vacua, of order $O\left(\bar{\mu}^{4}\right)$. In this case, we can use the thin wall approximation [31] to estimate the bounce action and obtain $S \sim(\bar{m} / \bar{\mu})^{8}$. Again, we can make it parametrically large when $\bar{\mu} \ll \bar{m}$.

\section{Supersymmetry preserving vacua}

So far we studied supersymmetry breaking vacua. In addition to these, the model also has supersymmetric vacua. Here, we will show that these supersymmetry preserving vacua can also be identified in the free magnetic dual description. Following [12], we look for a supersymmetric vacuum where meson fields get large expectation values. By the vacuum expectation value of $Y$ and $\hat{\Phi}$, dual quarks $\chi, \tilde{\chi}$ and $\rho, \tilde{\rho}$ become massive and can be integrated out. Also in the energy scale $E<h m_{z}, Z$ and $\tilde{Z}$ should be integrated out. Thus, we are left with the superpotential

$$
W=-h \bar{m}^{2} Y-h \bar{\mu}^{2} \hat{\Phi}+\left(N_{F}-N_{C}\right) \Lambda_{\text {eff }}^{3},
$$

where the last term is generated by nonperturbative dynamics of a pure $\mathrm{SU}\left(N_{F}-N_{C}\right)$ gauge theory. The low energy scale $\Lambda_{\text {eff }}$ after decoupling of dual quarks, is given 
by the matching conditions at the two mass scales $h Y$ and $h \hat{\Phi}$,

$$
\Lambda_{\text {eff }}^{3}=\langle h Y\rangle\langle h \hat{\Phi}\rangle^{N_{C} /\left(N_{F}-N_{C}\right)} \Lambda_{m}^{\left(2 N_{F}-3 N_{C}\right) /\left(N_{F}-N_{C}\right)} .
$$

Note that $Z$ and $\tilde{Z}$ are singlets for the gauge group and do not contribute to running of the gauge coupling. With the nonperturbative superpotential, $F$-term conditions for light field $Y$ and $\hat{\Phi}$ have solutions of the form

$$
\begin{aligned}
& \langle h \hat{\Phi}\rangle=\bar{m}^{\left(2\left(N_{F}-N_{C}\right)\right) / N_{C}} \Lambda_{m}^{\left(3 N_{C}-2 N_{F}\right) /\left(N_{F}-N_{C}\right)}, \\
& \langle h Y\rangle=\frac{\bar{\mu}^{2}}{\bar{m}^{\left(2\left(2 N_{C}-N_{F}\right)\right) / N_{C}}} \Lambda_{m}^{\left(3 N_{C}-2 N_{F}\right) / N_{C}} .
\end{aligned}
$$

Since $\langle h \hat{\Phi}\rangle \gg\langle h Y\rangle$ and the difference of the vacuum expectation value $\hat{\Phi}$ between supersymmetric vacua and supersymmetry breaking vacua is very large, compared to the height of supersymmetry breaking vacua, we can estimate the Euclidean action for the decay process by triangle approximation [30],

$$
S \sim \frac{\langle h \hat{\Phi}\rangle^{4}}{\bar{\mu}^{4}} \sim\left(\frac{\bar{m}}{\bar{\mu}}\right)^{4}\left(\frac{\Lambda_{m}}{\bar{m}}\right)^{4\left(3 N_{C}-2 N_{F}\right) / N_{C}} .
$$

The factor $3 N_{C}-2 N_{F}$ is always positive. Therefore, with the mass hierarchy $\bar{\mu} \ll \bar{m}$ and $\bar{m} \ll \Lambda_{m}$, we can make the action arbitrarily large, and thus make the metastable vacua arbitrarily long-lived. These conditions also allow us to ignore higher order correction to the Kähler potential.

\section{B. Gaugino and scalar masses}

With the explicit $R$-symmetry breaking by $m_{z}$, direct mediation of supersymmetry breaking happens. The standard model gauge group can be embedded into either the $\mathrm{SU}\left(N_{F}-N_{C}\right)_{F}$ or the $\mathrm{SU}\left(N_{C}\right)_{F}$ flavor symmetry which remains unbroken at low energy.

The gaugino masses are, in this case, given by the same formula in Eq. (8) with mass matrix $\mathcal{M}$ :

$$
\mathcal{M}=\left(\begin{array}{cc}
\hat{\Phi} & \bar{m} \\
\bar{m} & m_{z}
\end{array}\right) .
$$

Therefore

$$
m_{\lambda}=\frac{g^{2} \bar{N}}{(4 \pi)^{2}} \frac{h \bar{\mu}^{2}}{\bar{m}} \frac{m_{z}}{\bar{m}}+O\left(\frac{m_{z}^{2}}{\bar{m}^{2}}\right),
$$

with $g^{2}$ the gauge coupling constant of the standard model gauge interaction. The factor $\bar{N}$ is again $\bar{N}=N_{C}(\bar{N}=$ $N_{F}-N_{C}$ ) when we embed the standard model gauge group into $\mathrm{SU}\left(N_{F}-N_{C}\right)\left(\mathrm{SU}\left(N_{C}\right)\right)$.

Scalar masses are also obtained by two-loop diagrams. It is calculated to be

$$
m_{i}^{2}=2 \bar{N} C_{2}^{i}\left(\frac{g^{2}}{(4 \pi)^{2}}\right)^{2}\left(\frac{h \bar{\mu}^{2}}{\bar{m}}\right)^{2}+O\left(\frac{m_{z}^{4}}{\bar{m}^{4}}\right) .
$$

$C_{2}^{i}$ is a quadratic Casimir factor for a field labeled $i$. For having a similar size of gaugino and scalar masses, $m_{z} \sim$ $\bar{m} / \sqrt{\bar{N}}$ is required. It is possible to have this relation as long as $m_{z}<\bar{m}$ without destabilizing the metastable vacuum.

\section{Mass spectrum and the Landau pole problem}

We summarize the mass spectrum at the ISS vacuum here. The massless modes are the Goldstone boson, $\operatorname{Im}[\operatorname{Tr} \delta \hat{\chi}]$, and the fermionic component of $\operatorname{Tr} \delta \hat{\chi}$. The pseudomoduli $\hat{\Phi}$ and $\operatorname{Re}[\operatorname{Tr} \delta \hat{\chi}]$ have masses which are similar sizes to the gauginos, i.e., $O(100 \mathrm{GeV})$. Other component fields in the chiral multiplets $Y, Z, \tilde{Z}, \rho, \tilde{\rho}, \chi$, and $\tilde{\chi}$ have masses of $O(h \bar{m})$ or are eaten by the gauge/ gaugino fields.

Discussion of the Landau pole depends on a way of embedding of the standard model gauge group into flavor symmetries. We separately discuss two cases. We find that it is possible to avoid a Landau pole if we embed the standard model gauge group into the $\mathrm{SU}\left(N_{F}-N_{C}\right)_{F}$ flavor symmetry and take the dynamical scale and the mass parameter $\bar{m}$ to be large enough. We also comment on an alternative possibility that the $\mathrm{SU}\left(N_{C}\right)$ gauge theory above the scale $m$ is a conformal field theory (CFT). This possibility allows us to take the mass parameter $m$ and the dynamical scale $\Lambda$ to be much lower than the unification scale without the Landau pole problem.

\section{Embedding $\mathrm{SU}(3) \times \mathrm{SU}(2) \times \mathrm{U}(1)$ into $\mathrm{SU}\left(N_{F}-N_{C}\right)_{F}$}

In this case, the pseudomoduli $\hat{\Phi}$ is a singlet under the standard model gauge group, and thus it does not contribute to the beta function.

The beta function coefficients of the SU(3) gauge coupling are

$$
\begin{aligned}
b_{3}\left(\mu_{R}<h \bar{m}\right) & =-3, \\
b_{3}\left(h \bar{m}<\mu_{R}<\Lambda\right) & =-3+2 N_{F}-N_{C}, \\
b_{3}\left(\mu_{R}>\Lambda\right) & =-3+N_{C},
\end{aligned}
$$

where $\mu_{R}$ is a renormalization scale. Above the mass scale $m_{X}(\gg \Lambda)$, which is defined in Eq. (9), the theory should be replaced by a renormalizable theory, where it necessarily contains additional fields. Therefore, there are contributions from those fields above the scale $m_{X}$. The size of the contributions depends on a specific ultraviolet completion of the theory.

In order for the embedding to be possible, $N_{F}-N_{C} \geq$ 5 , and from the condition $N_{C}<N_{F}<\frac{3}{2} N_{C}$, we obtain

$$
2 N_{F}-N_{C}>20, \quad N_{C}>10 .
$$

There is a quite large contribution to the beta function. To avoid a Landau pole below the unification scale, $M_{\mathrm{GUT}} \sim$ $10^{16} \mathrm{GeV}$, the mass scales $h \bar{m}$ and $\Lambda$ should be high 
enough. For example, $\Lambda \sim M_{\mathrm{GUT}}$ and $h \bar{m} \geq 10^{13} \mathrm{GeV}$ can avoid the Landau pole.

Model in the conformal window.-Although it is not conclusive, the authors of Ref. [12] suggested that there is a metastable supersymmetry breaking vacuum also when the numbers of colors and flavors are the same. If it is the case, there is an interesting possibility that we can go into the conformal window, $\frac{3}{2} N_{C} \leq N_{F}<3 N_{C}$. If $N_{F}$ is in the conformal window, the gauge coupling of $\mathrm{SU}\left(N_{C}\right)$ flows into the conformal fixed point at some scale $\Lambda_{*}$. The theory stays as a CFT until the mass term $m\left(Q_{I} \bar{Q}_{I}\right)$ becomes important, and eventually at a lower scale $\Lambda \sim m$, the theory exits from the CFT and becomes strongly coupled. The effective theory below the scale $\Lambda \sim m$ is described by an $\mathrm{SU}\left(N_{C}\right)$ gauge theory with $N_{C}$ flavors with a mass term $\mu\left(Q_{a} \bar{Q}_{a}\right)$. This is exactly the ISS model with $N_{C}$ flavors. Once we assume the existence of the metastable supersymmetry breaking vacuum, direct gauge mediation should happen as we discussed in the previous section although we have lost the control of the perturbative calculation. (See [32] for a similar model.) The beta function coefficient $b_{3}$ is in this case,

$$
\begin{aligned}
b_{3}\left(\mu_{R}<\Lambda\right) & =-3, \\
b_{3}\left(\Lambda<\mu_{R}<\Lambda_{*}\right) & =-3+\frac{3 N_{C}^{2}}{N_{F}}+\Delta, \\
b_{3}\left(\mu_{R}>\Lambda_{*}\right) & =-3+N_{C}+\Delta^{\prime},
\end{aligned}
$$

where we have included a contribution from anomalous dimensions of $Q$ 's in CFTs [33-35]. The factors $\Delta$ and $\Delta^{\prime}$ are unspecified contributions from the fields which generate the $m_{z}$ term.

With $\frac{3}{2} N_{C} \leq N_{F}<3 N_{C}$ and $N_{F}-N_{C} \geq 5$, we find

$$
N_{C} \geq 3, \quad N_{F} \geq 8, \quad \frac{3 N_{C}^{2}}{N_{F}} \geq \frac{27}{8} .
$$

Therefore, the dynamical scale $\Lambda \sim m$ can be much lower than the unification scale in this case. For example, if we take the ultraviolet completion to be simply adding a pair of massive fields $\eta_{I a}$ and $\tilde{\eta}_{a I}$ which couple to $\left(Q_{a} \bar{Q}_{I}\right)$ and $\left(Q_{I} \bar{Q}_{a}\right)$, respectively, the additional contributions are $\Delta=$ $\Delta^{\prime}=N_{C}$. In this case, we can take the dynamical scale $\Lambda \sim m$ to be as low as $O(100-1000 \mathrm{TeV})$ without a Landau pole problem for $N_{C}=3$ and $N_{F}=8$.

We implicitly took the scale $m_{X}$, where the $m_{z}$ term is generated, to be $O(\Lambda)$ in Eq. (21) because of the requirement $m_{z} \sim \bar{m}$ for the sizes of the gaugino and scalar masses to be similar. With $m_{z} \sim \Lambda^{2} / m_{X}$ (see Eq. (9)) and $m \sim \Lambda$, we need to take $m_{X} \sim \Lambda$. However, the actual scale at which new fields appear can be much higher than $\Lambda$ or even $\Lambda_{*}$ when the anomalous dimensions of $Q$ and $\bar{Q}$ are large in the CFT. For example, when $N_{F} \leq 2 N_{C},\left(Q_{I} \bar{Q}_{a}\right) \times$ $\left(Q_{a} \bar{Q}_{I}\right)$ is a marginal or a relevant operator. In this case, it is not required to have an ultraviolet completion of the theory up to $O\left(\Lambda_{*}\right)$ or higher, i.e., $\Delta=0$, while satisfying $m_{z} \sim \bar{m}$. This can be understood by the running of the $1 / m_{X}$ parameter in the CFT:

$$
\frac{1}{m_{X}\left(\mu_{R}\right)}=\frac{1}{m_{X}(\Lambda)}\left(\frac{\mu_{R}}{\Lambda}\right)^{\left(2 N_{F}-6 N_{C}\right) / N_{F}} .
$$

The unspecified contribution $\Delta^{\prime}$ is not important if $\Lambda_{*}$ is high enough.

If $N_{F}-N_{C}>5$, there are flavors with mass $m$ which are not charged under the standard model gauge group. If we reduce the masses of those fields to be slightly smaller than $m$, the low energy effective theory below $\Lambda$ has more flavors and we can perform a reliable perturbative calculation of the potential for pseudomoduli.

It is interesting to note that this CFT model may be regarded as a dual description of models with a warped extra dimension in Refs. [36-39], where supersymmetry is broken on an infrared brane, and standard model gauge fields are living in the bulk of the extra dimension.

\section{Embedding $\mathrm{SU}(3) \times \mathrm{SU}(2) \times \mathrm{U}(1)$ into $\mathrm{SU}\left(N_{C}\right)_{F}$}

In this case, $b_{3}$ is given by

$$
\begin{aligned}
b_{3}\left(\mu_{R}<h \bar{m}\right) & =-3+N_{C}, \\
b_{3}\left(h \bar{m}<\mu_{R}<\Lambda\right) & =-3+2 N_{F}-N_{C}, \\
b_{3}\left(\mu_{R}>\Lambda\right) & =-3+N_{C} .
\end{aligned}
$$

The condition for the embedding to be possible is $N_{C} \geq 5$. Therefore

$$
2 N_{F}-N_{C}>5, \quad N_{C} \geq 5 .
$$

With this constraint, there is always a Landau pole below the unification scale. The situation does not improve even if we consider the possibility of the CFT above the mass scale $m$.

To summarize, by embedding the standard model gauge group in the $\mathrm{SU}\left(N_{F}-N_{C}\right)_{F}$ subgroup of the flavor symmetry, we can couple the ISS model to the standard model. The gaugino masses are generated at one loop, and the Landau pole problem can be avoided if the gauge coupling scale of the ISS sector is sufficiently high or if the theory above the mass scale $m$ is a CFT.

\section{ULTRAVIOLET COMPLETIONS}

The perturbation to the ISS model we considered in the previous section is nonrenormalizable in the electric description. In this section we will show that the model can be regarded as a low energy effective theory of a renormalizable gauge theory at high energy. Moreover, this renormalizable theory itself can be realized as a low energy effective theory on intersecting branes and on branes on a local Calabi-Yau manifold in string theory. In order to decouple Kaluza-Klein and string excitations from the gauge theory, the length scale of these brane configurations as well as the string length must be smaller than that of the 
gauge theory. These brane configurations are so simple that it may be possible to incorporate them in the ongoing effort to construct the minimal supersymmetric standard model from string theory compactifications.

One way to generate the nonrenormalizable interaction (9) is as follows. Consider an $\mathcal{N}=2$ quiver gauge theory with the gauge group $\mathrm{U}\left(N_{1}\right) \times \mathrm{U}\left(N_{2}\right) \times \mathrm{U}\left(N_{3}\right)$ with

$$
N_{1}=N_{F}-N_{C}, \quad N_{2}=N_{C}, \quad N_{3}=N_{C},
$$

and identify $\mathrm{U}\left(N_{2}\right)$ with the gauge group $\mathrm{U}\left(N_{C}\right)$ of the ISS model. ${ }^{4}$ We assume that the scales $\Lambda_{1}, \Lambda_{3}$ for the other gauge group factors are so low that we can treat $\mathrm{U}\left(N_{1}\right) \times$ $\mathrm{U}\left(N_{3}\right)$ as a flavor group. We then deform the theory by turning on the superpotential $W_{1}\left(X_{1}\right)+W_{2}\left(X_{2}\right)+W_{3}\left(X_{3}\right)$ for the adjoint fields $X_{1}, X_{2}, X_{3}$ in the $\mathcal{N}=2$ vector multiplets given by

$$
\begin{gathered}
W_{1}=\frac{M_{X}}{2} X_{1}^{2}+\alpha_{1} X_{1}, \quad W_{2}=-\frac{M_{X}}{2} X_{2}^{2}, \\
W_{3}=\frac{M_{X}}{2} X_{3}^{2}+\alpha_{3} X_{3} .
\end{gathered}
$$

This breaks $\mathcal{N}=2$ supersymmetry into $\mathcal{N}=1$, and the total tree-level superpotential of the deformed theory is

$$
\begin{aligned}
W_{\text {tree }}= & -Q_{21} X_{1} Q_{12}+Q_{12} X_{2} Q_{21}-Q_{32} X_{2} Q_{23} \\
& +Q_{23} X_{3} Q_{32}+W_{1}\left(X_{1}\right)+W_{2}\left(X_{2}\right)+W_{3}\left(X_{3}\right) .
\end{aligned}
$$

After integrating out massive fields $X_{i}$, the superpotential can be written as

$$
\begin{aligned}
W_{\text {tree }} & =\operatorname{Tr} m_{Q} Q \bar{Q}+\operatorname{Tr} K_{1} Q \bar{Q} K_{2} Q \bar{Q}, \\
m_{Q} & =\operatorname{diag}\left(\alpha_{1} / M_{X}, \alpha_{3} / M_{X}\right), \\
K_{1} & =\operatorname{diag}\left(0,1 / M_{X}\right), \\
K_{2} & =\operatorname{diag}(1,0) .
\end{aligned}
$$

This reproduces the interaction (9) and the mass terms for $\left(Q_{I}, Q_{a}\right)$ if we set

$$
\frac{\alpha_{1} \Lambda_{2}}{M_{X}}=h \bar{m}^{2}, \quad \frac{\alpha_{3} \Lambda_{2}}{M_{X}}=h \bar{\mu}^{2}, \quad \frac{\Lambda_{2}^{2}}{M_{X}}=h m_{z} .
$$

Since we suppose $\Lambda_{2}<M_{X}$, all the Eqs. (27) can be satisfied by appropriately choosing parameters $\alpha_{1,2}$ and $M_{X}$.

\section{A. Embedding in string theory}

In the perturbative string theory, the collective coordinates of D-branes are open strings ending on them [40]. Since the lightest degrees of freedom of open strings

\footnotetext{
${ }^{4}$ In the previous sections, we consider the case when the gauge group is $\mathrm{SU}\left(N_{C}\right)$. When the gauge group is $\mathrm{U}\left(N_{C}\right)$, the "baryon" symmetry is gauged and one of the pseudomoduli $\operatorname{Tr} \delta \hat{\chi}$ becomes massive at tree level due to the additional D-term condition. Otherwise, there is no major difference in properties of metastable vacua.
}

include gauge fields, a variety of gauge theories arise on intersecting branes in the low energy limit where the string length becomes small and the coupling of D-branes to the bulk gravitational degrees of freedom becomes negligible [41-43]. We will present an intersecting brane configuration where the deformed ISS model is realized as a low energy effective theory. One should not be confused that our use of the intersecting brane model implies that the theory above the dynamical scale $\Lambda$ is replaced by string theory or a higher dimensional theory. The string length and the compactification scale are much shorter than the gauge theory scale. It is one of the string miracles that quantum moduli spaces of low energy gauge theories are often realized as actual physical spaces such as brane configurations or Calabi-Yau geometry, allowing us to discuss deep infrared physics in the ultraviolet descriptions of the theories. This phenomenon has been well established for moduli spaces of supersymmetric vacua, and it has just begun to be explored for supersymmetry breaking vacua [44-49]. (For earlier works in this direction, see for example [50-52].) Here, we will find that metastable supersymmetry breaking vacua of the deformed ISS model are realized as geometric configurations of branes.

Consider type IIA superstring theory in the flat 10dimensional Minkowski spacetime with coordinates $x^{0, \ldots, 9}$. Introduce four NS5 branes located at $x^{7,8,9}=0$ and at different points in the $x^{6}$ direction, and extended in the $x^{0, \ldots, 3}$ and $x^{4,5}$ directions. Let us call these NS5 branes from the left to right along the $x^{6}$ direction as $\mathrm{NS}_{1}, \mathrm{NS}_{2}, \mathrm{NS}_{3}$, and $\mathrm{NS}_{4}$. We then suspend $\left(N_{F}-\right.$ $N_{C}$ ) D4 branes between $\mathrm{NS}_{1}$ and $\mathrm{NS}_{2}, N_{C}$ D4 branes between $\mathrm{NS}_{2}$ and $\mathrm{NS}_{3}$, and $N_{C}$ D4 branes between $\mathrm{NS}_{3}$ and $\mathrm{NS}_{4}$. The brane dynamics in the common $x^{0, \ldots, 3}$ directions is described by the $\mathcal{N}=2$ supersymmetric quiver gauge theory with the gauge group $\mathrm{U}\left(N_{F}-N_{C}\right) \times$ $\mathrm{U}\left(N_{C}\right) \times \mathrm{U}\left(N_{C}\right)$. Note that the gauge coupling constant $g_{\mathrm{YM}}^{(i)} i=1,2,3$ for the three gauge group factors is given at the string scale by

$$
\left(g_{\mathrm{YM}}^{(i)}\right)^{2}=g_{\mathrm{s}} \frac{\ell_{\mathrm{s}}}{L_{i}}
$$

where $g_{\mathrm{s}}$ and $\ell_{\mathrm{s}}$ are string coupling constant and string length, and $L_{1}, L_{2}, L_{3}$ are the lengths of the three types of D4 branes suspended between NS5 branes. The gauge couplings $g_{\text {YM }}^{(i=1,2,3)}$ set the initial conditions for the renormalization group equation at ultraviolet. The $\mathcal{N}=2$ quiver gauge theory is realized in the low energy limit where $g_{s}, \ell_{\mathrm{s}}, L_{i} \rightarrow 0$, keeping $g_{\mathrm{YM}}^{(i)}$ fixed. We choose $L_{2} \ll$ $L_{1}, L_{3}$ so that the gauge coupling constants for $\mathrm{U}\left(N_{1}\right) \times$ $\mathrm{U}\left(N_{3}\right)$ are small.

We can turn on the superpotentials $W_{1}+W_{2}+W_{3}$ by rotating $\mathrm{NS}_{2}$ and $\mathrm{NS}_{4}$ into the $x^{7,8}$ directions. More precisely, we use the complex coordinates $z=x^{4}+i x^{5}$ and $w=x^{7}+i x^{8}$ and rotate the two NS5 branes on the $z-w$ plane so that they are extended in the direction of 


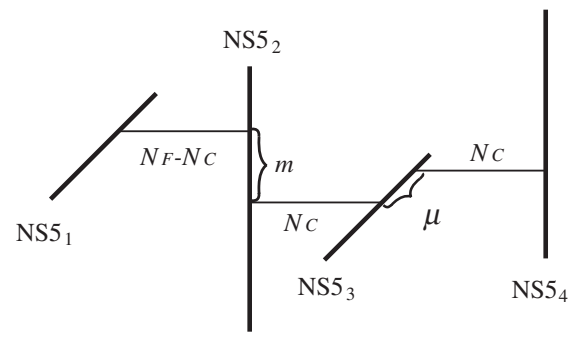

FIG. 2. The electric brane configuration.

$\cos \theta z+\sin \theta w$. The holomorphic rotation preserves $\mathcal{N}=$ 1 supersymmetry. In the field theory, this corresponds to turning on $W_{1}+W_{2}+W_{3}$ with $M_{X}=\tan \theta$ [53]. We can also turn on the quark masses $m$ and $\mu$ by moving NS5 $5_{1}$ and $\mathrm{NS}_{4}$ in the $w$ direction. The resulting configuration is shown in Fig. 2.

We can also T-dualize the NS5 branes to turn the D4 branes suspended between the NS5 branes into D-branes wrapping compact cycles in a local Calabi-Yau manifold [54]. Realizations of metastable vacua on branes partially wrapping cycles in Calabi-Yau manifolds have been discussed, for example, in [48,55].

The brane configuration shown in Fig. 2 is similar to the one that appeared recently in [47]. However, there are some important differences. In the model of [47], the quark masses $m$ and $\mu$ in the electric description are set equal to zero. Moreover the strong coupling scales of the three gauge group factors are chosen as $\Lambda_{1}, \Lambda_{2} \ll \Lambda_{3}$ in the model of [47], whereas $\Lambda_{1}, \Lambda_{3} \ll \Lambda_{2}$ in our model. These differences have led to different ways of supersymmetry breaking in these models. Despite the differences, some of the results in [47] may be useful for further studies of our model.

\section{B. Metastable supersymmetry breaking vacua on the brane configuration}

In [44,45], the ISS model and its magnetic dual were studied by realizing them on intersecting branes, and brane configurations for the supersymmetry breaking vacua were identified. The brane configurations provide a geometric way to understand the vacuum structure of the model. ${ }^{5}$ Recently it was used, for example, to study solitonic states on the metastable vacuum in the ISS model [56]. Here, we will present brane configurations that correspond to the metastable vacua in the deformed ISS model.

To identify the metastable vacua, we need to go to the magnetic description, which is realized on branes by exchanging $\mathrm{NS}_{2}$ and $\mathrm{NS}_{3}$. Since we assume $L_{2} \ll L_{1}, L_{3}$, it is reasonable to expect that the first duality transforma-

\footnotetext{
${ }^{5}$ See [46] on issues that arise when one turns on finite string coupling in these brane configurations. These issues are not relevant to our discussion below since we mostly deal with tree-level properties of type IIA superstring theory.
}

tion involves only these two NS5 branes. To avoid confusion, let us call the resulting NS5 branes $\mathrm{NS}_{1}, \mathrm{NS}_{2}^{\prime}$, $\mathrm{NS}_{3}^{\prime}$, and $\mathrm{NS}_{4}$ from the left to right in the $x^{6}$ direction. Note that $\mathrm{NS}_{1}$ and $\mathrm{NS}_{2}^{\prime}$ are parallel to each other, and so are $\mathrm{NS5}_{3}^{\prime}$ and $\mathrm{NS5}_{4}$. There are $\left(N_{F}-N_{C}\right)$ D4 branes between $\mathrm{NS}_{1}$ and $\mathrm{NS5}_{3}^{\prime}, N_{C}$ anti-D4 branes between $\mathrm{NS}_{2}^{\prime}$ and $\mathrm{NS}_{3}^{\prime}$, and $N_{C}$ D4 branes between $\mathrm{NS}_{2}^{\prime}$ and $\mathrm{NS}_{4}$.

The ISS vacuum is obtained by bending the $N_{C}$ D4 branes between $\mathrm{NS}_{2}^{\prime}$ and $\mathrm{NS}_{4}$ toward $\mathrm{NS}_{3}^{\prime}$, disconnecting each of them at $\mathrm{NS}_{3}^{\prime}$, and annihilating their segments between $\mathrm{NS5}_{2}^{\prime}$ and NS5 ${ }_{3}^{\prime}$ with the $N_{C}$ anti-D4 branes by the tachyon condensation. The resulting brane configuration is shown in Fig. 3. Note that this configuration breaks supersymmetry since the D4 branes between $\mathrm{NS}_{1}$ and $\mathrm{NS}_{3}^{\prime}$ and the D4 branes between $\mathrm{NS}_{3}^{\prime}$ and $\mathrm{NS}_{4}$ are in angles. Since their endpoint separation is of the order of $|m|$ whereas the supersymmetry breaking is of the order of their relative angles $\sim|\mu|$, an open string stretched between these D4 branes does not contain a tachyon mode provided $|m| \gg|\mu|$. Since $\mathrm{NS5}_{3}^{\prime}$ and $\mathrm{NS5}_{4}$ are parallel to each other, the $N_{C}$ D4 branes between them can move along them. This freedom corresponds to pseudomoduli $\hat{\Phi}$. These D4 branes are stabilized by a potential induced by closed string exchange between them and the D4 branes between $\mathrm{NS}_{1}$ and $\mathrm{NS5}_{2}^{\prime}$, which is the closed string dual of the Coleman-Weinberg potential.

We can also identify the other metastable vacua of the deformed ISS model. Let us take $n$ of the $N_{C}$ D4 branes between $\mathrm{NS}_{3}^{\prime}$ and $\mathrm{NS}_{4}$ and move them toward the $\left(N_{F}-\right.$ $\left.N_{C}\right)$ D4 branes between NS5 1 and NS5 $5_{3}^{\prime}$. Doing this costs energy since these D4 branes have to climb up the Coleman-Weinberg potential. Eventually, as they approach the D4 branes between $\mathrm{NS}_{1}$ and $\mathrm{NS}_{3}^{\prime}$, open strings between the two kinds of D4 branes start developing tachyonic modes. The tachyon condensation then reconnects $n$ pairs of $\mathrm{D} 4$ branes, leading to the brane configuration as shown in Fig. 4. This process lowers the vacuum energy since the length of the single D4 brane between $\mathrm{NS}_{1}$ and $\mathrm{NS}_{4}$ is shorter than the sum of the two D4 branes before the reconnection.

One can show that these brane configurations reproduce various features of the corresponding metastable vacua, such as their vacuum energies, expectation values of vari-

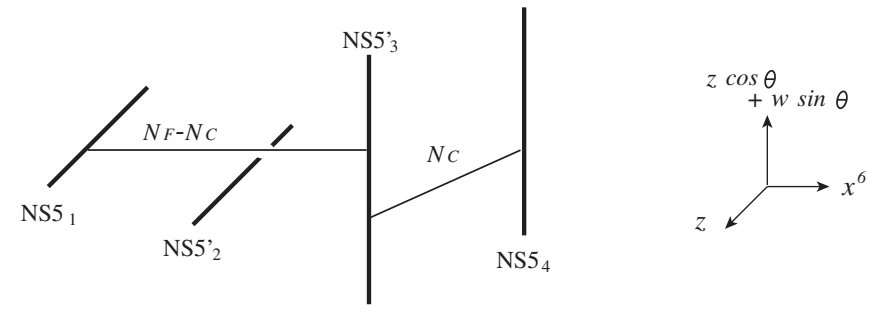

FIG. 3. The brane configuration for the ISS vacuum in the deformed ISS model. 

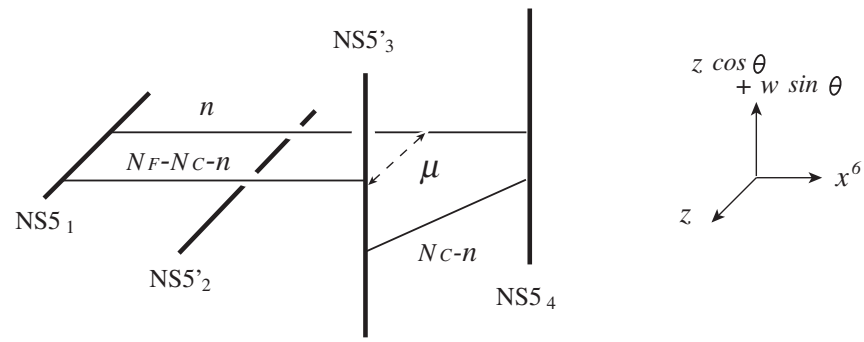

FIG. 4. The brane configuration for the metastable vacua with lower energies.

ous fields (such as $\rho \tilde{\rho}, Y$, and $\hat{\Phi}$ ), and their decay processes. This can be done by a straightforward application of the brane configuration analysis in [44-46], and we leave it as an exercise for the readers.

\section{METASTABILITY AT FINITE TEMPERATURE?}

It has been shown that the metastable supersymmetry breaking vacuum in the ISS model is favored in the thermal history of the Universe [26-28]. The essential observation is that there are more light degrees of freedom in the supersymmetry breaking vacuum compared to the super- symmetric one. Finite temperature effects make the metastable vacuum more attractive in this circumstance.

In the deformed ISS model we discussed in this paper, there are many other metastable vacua. However, interestingly, the desired vacuum (the ISS vacuum) possesses the largest symmetry group among those vacua. In other vacua, the number of degrees of freedom of the pseudomoduli is reduced because some components of $\hat{\Phi}$ have masses at tree level. Therefore, the desired vacuum is the most attractive in the thermal history of the Universe.

\section{ACKNOWLEDGMENTS}

We would like to thank K. Intriligator, H. Murayama, E. Silverstein, T. Watari, and T. Yanagida for discussions. R. K. thanks the hospitality of the high energy theory group at Rutgers University. The work of R. K. was supported by the U.S. Department of Energy under Contract No. DEAC02-76SF00515. The work of H.O. and Y.O. is supported in part by the U.S. Department of Energy under Contract No. DE-FG03-92-ER40701. H. O. is also supported in part by the U.S. National Science Foundation under Contract No. OISE-0403366. Y. O. also acknowledges support by JSPS.
[1] S. Dimopoulos and H. Georgi, Nucl. Phys. B193, 150 (1981).

[2] S. Dimopoulos, S. Raby, and F. Wilczek, Phys. Rev. D 24, 1681 (1981).

[3] N. Sakai, Z. Phys. C 11, 153 (1981).

[4] M. Dine, W. Fischler, and M. Srednicki, Nucl. Phys. B189, 575 (1981).

[5] S. Dimopoulos and S. Raby, Nucl. Phys. B192, 353 (1981).

[6] E. Witten, Nucl. Phys. B202, 253 (1982).

[7] I. Affleck, M. Dine, and N. Seiberg, Phys. Rev. Lett. 51, 1026 (1983).

[8] I. Affleck, M. Dine, and N. Seiberg, Nucl. Phys. B241, 493 (1984).

[9] I. Affleck, M. Dine, and N. Seiberg, Nucl. Phys. B256, 557 (1985).

[10] E. Poppitz and S. P. Trivedi, Phys. Rev. D 55, 5508 (1997).

[11] N. Arkani-Hamed, J. March-Russell, and H. Murayama, Nucl. Phys. B509, 3 (1998).

[12] K. Intriligator, N. Seiberg, and D. Shih, J. High Energy Phys. 04 (2006) 021

[13] T. Banks, hep-ph/0606313.

[14] M. Dine and J. Mason, hep-ph/0611312.

[15] E. Witten, Phys. Lett. B 105, 267 (1981).

[16] H. Murayama, Phys. Rev. Lett. 79, 18 (1997).

[17] S. Dimopoulos, G. R. Dvali, and R. Rattazzi, Phys. Lett. B 413, 336 (1997)

[18] M. A. Luty, Phys. Lett. B 414, 71 (1997).

[19] K. Agashe, Phys. Lett. B 435, 83 (1998).
[20] R. Kitano, Phys. Lett. B 641, 203 (2006).

[21] K. I. Izawa, Y. Nomura, K. Tobe, and T. Yanagida, Phys. Rev. D 56, 2886 (1997).

[22] R. Kitano, Phys. Rev. D 74, 115002 (2006).

[23] M. Dine and W. Fischler, Phys. Lett. B 110, 227 (1982).

[24] C. R. Nappi and B. A. Ovrut, Phys. Lett. B 113, 175 (1982).

[25] L. Alvarez-Gaume, M. Claudson, and M. B. Wise, Nucl. Phys. B207, 96 (1982).

[26] S. A. Abel, C. S. Chu, J. Jaeckel, and V. V. Khoze, hep-th/ 0610334.

[27] N. J. Craig, P. J. Fox, and J. G. Wacker, hep-th/0611006.

[28] W. Fischler, V. Kaplunovsky, C. Krishnan, L. Mannelli, and M. Torres, hep-th/0611018.

[29] A. E. Nelson and N. Seiberg, Nucl. Phys. B416, 46 (1994).

[30] M. J. Duncan and L. G. Jensen, Phys. Lett. B 291, 109 (1992).

[31] S. R. Coleman, Phys. Rev. D 15, 2929 (1977); 16, 1248(E) (1977).

[32] K. I. Izawa and T. Yanagida, Prog. Theor. Phys. 114, 433 (2005).

[33] V. A. Novikov, M. A. Shifman, A. I. Vainshtein, and V.I. Zakharov, Nucl. Phys. B223, 445 (1983).

[34] M. A. Shifman and A. I. Vainshtein, Nucl. Phys. B277, 456 (1986); Zh. Eksp. Teor. Fiz. 91, 723 1986) [Sov. Phys. JETP 64, 428 (1986].

[35] N. Seiberg, Phys. Rev. D 49, 6857 (1994).

[36] T. Gherghetta and A. Pomarol, Nucl. Phys. B586, 141 (2000). 
[37] T. Gherghetta and A. Pomarol, Nucl. Phys. B602, 3 (2001).

[38] W. D. Goldberger, Y. Nomura, and D. R. Smith, Phys. Rev. D 67, 075021 (2003).

[39] Y. Nomura, hep-ph/0410348.

[40] J. Polchinski, Phys. Rev. Lett. 75, 4724 (1995).

[41] A. Hanany and E. Witten, Nucl. Phys. B492, 152 (1997).

[42] S. Elitzur, A. Giveon, and D. Kutasov, Phys. Lett. B 400, 269 (1997).

[43] A. Giveon and D. Kutasov, Rev. Mod. Phys. 71, 983 (1999).

[44] H. Ooguri and Y. Ookouchi, Phys. Lett. B 641, 323 (2006).

[45] S. Franco, I. Garcia-Etxebarria, and A. M. Uranga, hep-th/ 0607218.

[46] I. Bena, E. Gorbatov, S. Hellerman, N. Seiberg, and D. Shih, J. High Energy Phys. 11 (2006) 088.
[47] R. Argurio, M. Bertolini, S. Franco, and S. Kachru, hep-th/ 0610212.

[48] M. Aganagic, C. Beem, J. Seo, and C. Vafa, hep-th/ 0610249.

[49] H. Verlinde, hep-th/0611069.

[50] J. de Boer, K. Hori, H. Ooguri, and Y. Oz, Nucl. Phys. B522, 20 (1998).

[51] C. Vafa, J. Math. Phys. (N.Y.) 42, 2798 (2001).

[52] S. Kachru, J. Pearson, and H. L. Verlinde, J. High Energy Phys. 06 (2002) 021

[53] J. L. F. Barbon, Phys. Lett. B 402, 59 (1997).

[54] F. Cachazo, B. Fiol, K. A. Intriligator, S. Katz, and C. Vafa, Nucl. Phys. B628, 3 (2002).

[55] H. Ooguri and Y. Ookouchi, Nucl. Phys. B755, 239 (2006).

[56] M. Eto, K. Hashimoto, and S. Terashima, hep-th/0610042. 